\title{
What can we learn from over 100,000 Escherichia coli genomes?
}

2

3

$4{ }^{1}$ Department of Biomedical Informatics, University of Arkansas for Medical Sciences, Little

5 Rock, Arkansas, USA

$6{ }^{2}$ The Bredesen Center for Interdisciplinary Research and Graduate Education, University of

7 Tennessee, Knoxville, TN, USA

$8{ }^{3}$ Department of Electrical Engineering and Computer Science, University of Tennessee,

9 Knoxville, TN, USA

$10{ }^{4}$ Molecular Microbiology and Genomics Consultants, Zotzenheim, Germany

12 \#Corresponding author: DWUssery@uams.edu

$14 *$ These authors contributed equally

16 Running title: Insights from over 100,000 E. coli genomes

18 Keywords: Escherichia coli, phylogroup, pangenome, core genes, comparative genomics, 19 population structure, taxonomy, Mash, phylotype, Shigella, phylogeny, phylotype 


\section{ABSTRACT}

The explosion of microbial genome sequences in public databases allows for largescale population genomic studies of bacterial species, such as Escherichia coli. In this study, we examine and classify more than one hundred thousand $E$. coli and Shigella genomes. After removing outliers, a semi-automated Mash-based analysis of 10,667 assembled genomes reveals 14 distinct phylogroups. A representative genome or medoid identified for each phylogroup serves as a proxy to classify more than 95,000 unassembled genomes. This analysis shows that most sequenced $E$. coli genomes belong to 4 phylogroups (A, C, B1 and E2(O157)). Authenticity of the 14 phylogroups described is supported by pangenomic and phylogenetic analyses, which show differences in gene preservation between phylogroups. A phylogenetic tree constructed with 2,613 single copy core genes along with a matrix of phylogenetic profiles is used to confirm that the 14 phylogroups change at different rates of gene gain/loss/duplication. The methodology used in this work is able to identify previously uncharacterized phylogroups in $E$. coli species. Some of these new phylogroups harbor clonal strains that have undergone a process of genomic adaptation to the acquisition of new genomic elements related to virulence or antibiotic resistance. This is, to our knowledge, the largest $E$. coli genome dataset analyzed to date and provides valuable insights into the population structure of the species.

E. coli is a common inhabitant of the gastrointestinal tract of warm-blooded organisms, and can also be found in soil and freshwater environments ${ }^{1}$. The species is comprised of both commensal and pathogenic strains which can cause disease in a wide variety of hosts. In humans, pathogenic E. coli strains are a leading cause of diarrhea-associated hospitalizations ${ }^{2}$. Some of the reasons why E. coli is intensely studied are: rapid growth rate in the presence of oxygen, easy adaptation to environmental changes, and the relative ease with which it can be genetically manipulated ${ }^{3}$. Genomic diversity of the species, to which the genus Shigella has been proposed to be included ${ }^{4,5}$, is reflected by the existence of several phylogenetic groups (phylogroups) that have been identified using a variety of different methods ${ }^{6-8}$.

Historically, four phylogroups have been recognized as detected by triplex PCR: A, B1, $\mathrm{B} 2$, and $\mathrm{D}^{6,8}$ and three more were added later ${ }^{9}$ : phylogroups $\mathrm{C}$ (closest relative to $\mathrm{B} 1$ ), $\mathrm{F}$ (as a 
49 sister group of phylogroup B2), and E to which many D members were reassigned. Some studies

50 have further subdivided these phylogroups with subdivisions of $\mathrm{F}$ and $\mathrm{D}$, and separate

51 phylogroups for Shigella species ${ }^{10}$. Recently, Clermont et al. ${ }^{11}$ characterized phylotype G using

52 multiplex PCR as an intermediate phylogroup between B2 and F. These phylogroups are thought

53 to be monophyletic ${ }^{8,10}$ and partially coincide with different ecological niches and lifestyles.

54 Moreover, phylogroups differ in metabolic characteristics, the presence of virulence genes, and

55 also in antibiotic resistance profiles ${ }^{8,12-14}$.

56 Here we describe a comprehensive analysis of over 100,000 publicly available genome

57 sequences, consisting of 12,602 assembled genomic sequences from GenBank and over 125,000

58 unassembled genome sequences from the Sequence Read Archive (SRA). This study combines

59 whole genome sequences (WGS) and SRA unassembled genomes using high-performance

60 computing resources to conduct, to our knowledge, the largest analysis to date of the population

61 structure of E. coli. We have assessed the genomic similarities and differences between

62 phylogroups to characterize the genetic heterogeneity of these different phylogenetic lineages.

63 We have also identified 14 'medoid'"15 genomes that can be considered as the genetic 'center' of

64 each of the phylogroups in our dataset and can be used as a representative sequence for the

65 associated phylogroup. Furthermore, this study has application to the fields of public health and

66 medical science as it provides detailed information of the existing diversity of the $E$. coli species

67 enabling public health researchers to identify pathogenic strains that belong to the same genetic

68 lineage appearing in outbreaks at different temporal and geographical locations.

\section{RESULTS}

71 Mash analysis of E. coli genomic sequences reveals 14 phylogroups. As illustrated by Fig. 1,

72 Mash-based clustering methodology differentiated 14 different phylogroups consisting of E. coli:

73 G, B2-1, B2-2, F, D1, D2, D3, E2(O157), E1, A, C, B1, and Shigella: Shig1 and Shig2 (ordered

74 as in Fig. 1) by using a cutoff in which the last literature accepted phylogroup became visible.

75 The phylogroups Shig1 and Shig2 exclusively contained Shigella species, but Shigella sp.

76 genomes were also found in phylogroups A, B1, B2-2, D2, D3, E1, and F (Supplementary Figure

77 1). Genomes within each of these phylogroups share a lower intragroup distance (meaning higher 
78 genetic similarity) than they do to any other genome within the rest of the species. In addition,

79 the genetic relatedness between any phylogroup and the rest of the species is graphically shown.

80 For example, phylogroups A, B1, and C are more closely related to each other than any one of

81 these phylogroups are to B2-1 or B2-2, as illustrated by lower Mash distances between

82 phylogroups A, B1, and C compared to B2-1 or B2-2. Fig. 1 also illustrates the phylogroup

83 substructure or intragroup genetic relatedness. E2(O157), Shig1, and Shig2 harbor the most

84 homogeneous genomes, which can be seen in the limited range of Mash distances within these

85 phylogroups. On the other hand, B1 and B2-2 are more heterogenous as shown by numerous

86 smaller dark teal squares that correspond to clusters of genomes that have a lower Mash distance

87 between them compared to the rest of the genomes in that phylogroup. The relative abundance of

88 phylogroup sequences with respect to each other can also be observed in Fig. 1. G has the

89 smallest number of genomes sequenced and B1 has the largest number of sequenced genomes in

90 the assembled dataset.

91 Microreact ${ }^{16}$ was utilized to further explore the results of the Mash-based analysis, as this

92 provides an easy medium for researchers to determine the closest genetic neighbors to any

93 genome in this dataset. Additionally, due to the inclusion of some clinically relevant outbreak

94 strains, such as $\mathrm{O} 157: \mathrm{H} 7, \mathrm{O} 104: \mathrm{H} 4$, and $\mathrm{O} 104: \mathrm{H} 21$, basic retroactive genomic surveillance is

95 possible by identifying strains of known outbreaks and noting their nearest neighbors. This data

96 is available on Microreact at: https://microreact.org/project/10667ecoli/4098eb8c.

98 Currently sequenced $E$. coli and Shigella species can be represented by 14 medoid genomes.

99 We were able to determine that 14 representative genomes can serve as the medoid or the

100 "genomic center" of each phylogroup based on the 10,667 analyzed genomes. Our results show

101 high correspondence with the recently proposed evolutionary scenario for the E. coli species $^{17}$

102 (Fig. 2). The Cytoscape analysis showed that the two B2 phylogroups are the most genetically

103 distinct from the remainder of the species as they separate earliest from the other phylogroups.

104 At the final Mash value cutoff of 0.0095, the C and B1 phylogroups become the last two groups

105 to separate. This last split is indicative of the relatively large shared genomic content between

106 these two phylogroups. The resultant Cytoscape graphs were collected into a video available as

107 Supplementary Video 1, and a collection of stills is available on the service figshare via 
108

109

110

111

112

113

114

115

116

117

118

119

120

121

122

123

124

125

126

127

128

129

130

131

132

133

134

135

136 http://dx.doi.org/10.6084/m9.figshare.11473308. Between the initial Cytoscape frame and the final frame, the number of genomes represented decreased by $43 \%$ while the edges (connections between genomes and medoids) decreased by $96 \%$. As the cutoff decreases, some genomes are no longer represented in the Cytoscape analysis due to having no Mash distance equal to or less than the applied cutoff. As expected, the overall interconnectivity between the different phylogroups drops significantly with the cutoff, but intraconnectivity within the phylogroups does not. Upon visualization and inspection of the data via Cytoscape, we could verify that each medoid is representative of its entire phylogroup and therefore the 14 medoids are suitable to be used for decreasing visual complexity without sacrificing accuracy. Information about the 14 found medoids is available in Supplementary Table 2.

Most sequenced E. coli genomes belong to 4 phylogroups. The use of medoid genomes as a proxy to classify more than 100,000 genomes revealed that most of the currently sequenced $E$. coli strains belong to 4 phylogroups. Around two-thirds (67\%) of the analyzed SRA reads were predicted to belong to four phylogroups: A (23\%), C (15\%), B1 (15\%), and E2(O157) (14\%).

This large disparity in phylogroup diversity in the SRA dataset most likely reflects the research interests of the scientific and medical communities. Strains belonging to phylogroups B1, C, and E2(O157) are often pathogenic and of interest to medical research, while phylogroup A includes strains frequently used in the laboratory (e.g., strain K-12) or genetically modified strains (such as strains BL21 and REL606). Similarly, a little over two-thirds (70\%) of the 10,667 assembled genomes also belong to four phylogroups: B1 (28\%), A (21\%), B2-2 (13\%) and Shig2 (8\%). However, in the assembled genomes dataset, phylogroup $\mathrm{C}$ is only about 5\% and E2(O157) is about $7 \%$. It is somewhat unexpected that the assembled genomes have a different distribution of genomes than the unassembled dataset; however, this could be due to how fast and inexpensive unassembled genomes are to produce and their utility in genomic surveillance of outbreaks. A breakdown of the results for the SRA analysis including the number of medoid hits below the cutoff is summarized in Supplementary Table 3. Additionally, a collection of heatmaps with different membership cut-offs, ranging from one to 14 phylogroups can be found in Supplementary Figure 2. 
137 Members of Mash phylogroups possess different genomic features. Since Mash values provide

138 a measure of similarity via distance between pairs of genomes, the phylogroups of Fig. 1 are the

139 consequence of differences/similarities in the genetic content of each genome with respect to the

140 rest of the genomes included in the analysis. Differences in genome size and percentage of GC

141 content between phylogenetic groups were observed (Supplementary Figure 3) and statistical

142 tests were performed by ANOVA and Tukey's multiple comparison test (see Methods and

143 Supplementary Table 4). According to these analyses, genomes from phylogroups Shig1, Shig2,

$144 \mathrm{~A}, \mathrm{~B} 1$ and B2-1 are significantly smaller in size than phylogroups $\mathrm{E} 2(\mathrm{O} 157)$ and $\mathrm{C}(\mathrm{P}<0.01)$.

145 The smaller genome size of the strains from both Shigella phylogroups is indicative of a 146 reductive evolution of the genomes of these strains as previously described ${ }^{18}$ by Weinert and

147 Welch which is mainly driven by their role as intracellular pathogens. Other enteroinvasive $E$.

148 coli strains such as serotypes O124, O152, O135 and O112ac were classified inside phylogroups

149 A (typically engineered, lab, and commensal strains) and B1 (often environmental strains) which

150 are the most heterogeneous phylogroups due to the diverse nature of their strains in terms of their

151 environmental niche. This heterogeneity is also reflected in the large ranges of genome size and

152 GC content of these two phylogroups. However, reduced genome size is not associated with

153 pathogenicity per se, as the large genomes of E2(O157) and C phylogroups illustrate. Larger

154 genome sizes associated with virulence may result from the accumulation of virulence genes in

155 prophages, pathogenicity islands, and plasmids ${ }^{19}$. Significant genomic differences in GC content,

156 with respect to other phylogroups were only found for the two Shigella phylogroups $(\mathrm{P}<0.01)$,

157 which also agrees with an ongoing purifying or negative selection occurring in these genomes ${ }^{18}$.

158 These characteristics might reflect the different evolutionary strategies and opposite selection

159 pressures as a consequence of adaptation to diverse niches in which the different phylogroups

160 have evolved ${ }^{20}$.

161 Level of preservation of homologous genes varies between phylogroups. To evaluate the

162 existence of functional traits associated with each of the phylogroups, we conducted pangenome-

163 approach based analyses using the proteomes of the 10,667 assembled genomes. In addition,

164 separate pan and core genomes were calculated for the 14 individual phylogroups. This approach

165 allows us to highlight the unique proteomic cores of each phylogroup, which in turns helps to 
166 define their distinct biology. The total set of genes of the species (pangenome) is comprised of 167 135,983 clusters of homologous proteins (Table 1). By testing the cutoffs for core genome 168 conservation from $90 \%$ to $99 \%$ of the genomes (Supplementary Fig. 4) we concluded that, while 169 the traditional cutoff for core genome calculation of $95 \%$ of genomes would suffice, a cutoff of $17097 \%$ can minimize erroneous false positive core genes thus providing a more stringent result.

171 Therefore, we defined the core genome as homologous genes shared by at least $97 \%$ of the 172 genomes ( ${ }^{\mathrm{TOT}}$ core97), which produced a core genome of 2,663 clusters $(1.96 \%$ of the total 173 pangenome clusters). The ${ }^{\mathrm{TOT}}$ core 97 , colored green in Fig. 3a, contains the well-preserved genes 174 that define the species, and for the shortest sequenced genomes (e.g. Escherichia coli str. K-12 175 substr. MDS42, phylogroup A), these constitute approximately 74\% of their gene content; in 176 contrast, for the largest genomes (e.g. E. coli Ec138B_L1, phylogroup A) this fraction is only 177 about $32 \%$.

By defining phylogroup-specific core genomes ( $\left.{ }^{\mathrm{PHY}} \mathrm{core}_{97}\right)$ it becomes apparent that large differences exist between the levels of gene preservation for each of the phylogroups (Fig. 3a). Predictably, the phylogroup with the largest number of ${ }^{\mathrm{PHY}}$ core $_{97}$ gene clusters is E2(O157). Not only do its members have large genomes, but this phylogroup is also very homogeneous as it

182 mostly contains $E$. coli $\mathrm{O} 157: \mathrm{H} 7$ strains that have a clonal origin ${ }^{21}$. Relatively large ${ }^{\mathrm{PHY}}$ core $_{97}$ are 183 also observed for phylogroups C, harboring strains of clinically relevant non-O157 enterohemorrhagic (EHEC) serotypes such as O111 and O26, and for phylogroup Shig2, whose members have relatively short genomes as it is mainly composed of $S$. sonnei strains, suggesting that these phylogroups are relatively homogeneous which increases the size of the core genome in turn decreasing the fraction of accessory genes. At the other end of the spectrum, the phylogroup with the smallest core genome is Shig1 followed by phylogroups B1, E1, and A (Table 1). The small core genome of Shigl is related to its small genome size, while more numerous phylogroups A, E1, and B1 contain more diverse members, resulting in a larger

191 fraction of accessory genes and a smaller phylogroup-specific core. This observation concurs 192 with the tendency of other environmental strains that usually present open pangenomes with 193 higher ratios of accessory and unique genes ${ }^{22,23}$. Nevertheless, although Shig1 phylogroup has 194 the smallest number of core genes, this number represents almost $29 \%$ of the total clusters found 
195 in this phylogroup (Table 1), which is the highest ratio of core gene clusters per phylogroup196 specific pangenome of the analysis. Phylogroups with fewer members can also produce larger 197 core genome fractions with respect to their pangenome due to sampling biases. Phylogroup G 198 was recently described by Clermont et al. ${ }^{11}$ as a multidrug resistant extra-intestinal pathogenic 199 phylogroup (ExPEC). G strains are closely related to strains from the B2 complex, and are 200 commonly isolated from poultry and poultry meat products, which coincides with our analyses 201 and available metadata. Although phylogroup $\mathrm{G}$ has the fewest number of strains in our dataset, 202 we believe that the high core/pan ratio of this phylogroup is due to the overabundance of the 203 sequence type ST117 (79\% of the strains) which makes this phylogroup quite homogeneous. 204 Based on these observations we conclude that the relative ratio of ${ }^{\mathrm{PHY}}$ core 97 to the total 205 phylogroup pangenome clusters is a measure of the intragroup diversity.

206 To analyze the distribution of the 14 phylogroups in terms of their shared genetic content,

207 a two-dimensional projection of the presence or absence of all protein families (complete 208 pangenome) for the 10,667 assembled genomes was represented by a Principal Coordinate 209 Analysis (PCoA) as shown in Fig. 3b. An initial observation of the PCoA plot is that 210 phylogroups segregated on the left side of the Y axis (B2-1, B2-2, G, F, D1, D2, D3) comprise 211 phylogroups that contain large numbers of strains labeled as extra-intestinal E. coli strains 212 (ExPEC) ${ }^{11,13,24}$. The observed overlap of B2-1 with the B2-2 phylogroup in Fig. 3b could be due 213 to their shared evolutionary history. For example, in silico MLST analyses shows that at least $21480 \%$ of B2-1 strains belong to the sequence type ST131, a multidrug resistant clonal group of 215 ExPEC that recently emerged from the B2-2 phylogroup ${ }^{25}$. This explains the high degree of 216 homogeneity of B2-1 phylogroup. Moreover, strains characterized as ST131 were not found in 217 other phylogroups in our dataset. It appears that the rapid and differential acquisition of unique 218 virulence and mobile genetic elements by ST131 strains ${ }^{26}$ make it possible to discriminate 219 between B2-1 (mainly ST131 strains) and B2-2 phylogroups using WGS approaches such as the 220 one used in this work.

221 While most of the phylogroups seem to have a relatively horizontal distribution within 222 the PCoA plot, phylogroups E2(O157) and Shig2 show the most striking differences in regards 223 to their vertical distribution with respect to the rest of phylogroups. As commented before, Shig2 
224 and E2(O157) are very homogeneous phylogroups, with large ${ }^{\mathrm{PHY}}$ core 97 that contain over 1,000

225 more protein families than the ${ }^{\mathrm{TOT}}$ core $_{97}$ of the species. These phylogroup-specific core genes

226 could contain genetic signatures that are not present in the core genome of other phylogroups,

227 and therefore would confer to all phylogroup members with intrinsic and distinguishable traits

228 making them "traceable" in terms of genetic content from the rest of phylogroups.

229 To represent the existence of unique phylogroup-specific core genes we made a 230 comparison only considering the $14{ }^{\mathrm{PHY}}$ core $_{97}$ and re-clustered them using the same parameters

231 as in the previous pangenome analyses. Fig. 3c is a representation of the sorted resultant clusters, 232 placing clusters from the ${ }^{\mathrm{TOT}}$ core $_{97}$ first, followed by the ${ }^{\mathrm{PHY}}$ core 97 clusters from the rest of 233 phylogroups. Sorting the clusters in this way, highlights clusters of core genes that are exclusive 234 to the ${ }^{\mathrm{PHY}}$ core 97 of a given phylotype. As can be observed, phylogroups E2(O157) and Shig2 235 possess the highest proportion of unique core genes (protein family clusters (columns) colored in 236 purple that are not present in the other phylogroups), followed by C, B2-1, and Shig1 237 phylogroups. Well-defined phylogroup unique core genes were also found for phylogroups D3 238 (uropathogenic multidrug resistant strains, mainly ST405 and ST38) and D1 (uropathogenic 239 multidrug resistant strains, predominantly ST69). A list of the phylogroup unique core genes 240 found and represented in Fig. 3c along with their associated functional features can be found in

241 Supplementary Table 5. Some of these clusters of genes comprise interesting characteristics such

242 as: a unique set of genes for synthesis of flagella only present in all strains belonging to the $\mathrm{C}$ 243 phylogroup, a complete set of genes for the transport of iron and ribose present in all members of 244 phylogroup E2(O157), and a set of genes for the synthesis of siderophores in B2-1 phylogroup

245 (Supplementary Table 5). The presence of unique-core gene clusters belonging to the ${ }^{\mathrm{PHY}} \mathrm{core}_{97}$ 246 of most phylogroups supports the existence of 14 distinguishable phylogroups within the species.

247 These genetic signatures might also have applications in public health as they could be utilized 248 for typing purposes.

249 However, not all phylogroups harbor phylogroups-specific genes. Phylogroups A and B1 250 have the weakest unique core signatures observed (along with D2 and E1 phylogroups), which 251 could be explained by the heterogeneous nature of both phylogroups. Although B1 is comprised 252 of strains isolated from environmental sources, it also contains enteropathogenic strains (EPEC), 253 EIEC strains and most of the Shigella strains, such as S. boydii and S. dysenteriae, that were not 
254 classified by Mash analysis in Shig1 or Shig2 phylogroups (Supplementary Fig. 1 and 255 Microreact data). These Shigella strains can be observed in the PCoA plot as the B1 small cluster 256 just on top of the Shig1 cluster. It is interesting to note that, although phylogroups A and B1 are 257 well-defined and described phylogroups, they are also considered as sister phylogroups with a 258 shared evolutionary history ${ }^{7,13,27}$ which is represented by their partial overlap observed in Fig. $3 b$ 259 and their late segregation observed in the Supplementary Video 1 and Fig. $2 \mathrm{~b}$ at a Mash distance 260 of 0.0115.

261 Phylogroups evolve with different gain/loss rates of protein families. Since the medoids were

262 shown to be suitable representative entities of the 14 phylogroups and the ${ }^{\mathrm{TOT}} \mathrm{core}_{97}$ genome was 263 established, a robust phylogeny analysis could now be performed based on the concatenated 264 independent alignment of 2,613 ${ }^{\mathrm{TOT}}$ core97 gene clusters without paralogs and a maximum 265 likelihood approach (Fig. 4a). The obtained phylogenetic tree, along with a matrix containing the 266 number of homolog genes per protein family for each representative genome, were used to 267 measure family sizes and lineage specific events applying an optimized gain-loss-duplicated 268 model. Differences in gene content between the medoids lead to the observation that the different 269 phylogroups have evolved with different gain/loss/duplication rates of protein families (Fig. 4b). 270 Relatively high ratios of gene expansion were observed for phylogroups Shig1, Shig2, C, and B2-1. As expected due to their smaller genomes, Shig1 and Shig2 possess the highest ratios of 272 gene loss, while Shig1, C, and Shig2 have the highest rates of gene duplication. On the other 273 hand, phylogroups A, B1, D3, and F have the lowest rates of gene gain, indicating these phylogroups have undergone limited gene expansion. It is also interesting to note is that

275 phylogroups D2, B1, and G have much lower rates of gene duplication compared to the other 276 phylogroups. In short, all phylogroups showed differential gain/loss duplication ratios of gene 277 families, even those that share a presumed ancestral history, such as the D phylogroups. As 278 stated before, D1 and D3 phylogroups comprise mainly UPEC strains and they are mainly 279 represented by one or two predominant sequence types. Conversely, D2 strains are typically 280 isolated from non-human sources with a large variation of sequence types. 


\section{Discussion}

284

285

286

287

288

289

290

291

292

293

294

295

296

297

298

299

300

301

302

303

304

305

306

307

308

309

310

311

Mash-based analysis provides a fast and highly scalable K-mer based approach that can be used on extremely large sets of genomes. Based on more than one hundred thousand genomes, the population structure of E. coli species appears to be more diverse than currently thought. The methodology applied here detected 14 phylogroups with a remarkably unequal distribution of membership in regards to the number of genomes per phylogroup. The current bias in sequencing data decreases the probability of finding the genetic signatures that captures the relative homogeneity of all members of the phylogroups. As a consequence, less numerously represented phylogroups may actually contain additional, as yet unidentified phylogroups or substructures within them and currently conclusions about their open or closed nature cannot be accurately drawn.

The presence of multiple phylogroups that share pathogenic characteristics and even share equivalent environmental niches, such as the D and B2 phylogroups, is indicative of faster evolutionary forces related to the pathogenic lifestyle of these strains that could be driven by the acquisition of virulence factors, recombinations, and interactions with the local flora of the host. While the analysis of gain/loss/duplication rates of the phylogroups does not assess the rate of mutation, the k-mer based Mash analysis can capture subtle differences in sequence similarity making these forces traceable. According to our analysis, the emergence of new phylogroups of E. coli is due to the pathogenic specialization of previously established phylogroups, such as phylogroups B2-1, D1, D2, and D3. These phylogroups could have acquired new genetic material causing the rest of the genome to adapt thus producing changes that are detected by WGS techniques such as Mash but are not detected by more target-restricted methods such as PCR. We therefore conclude that the use of WGS data with Mash to assess a bacterial species' genetic sub-structure is essential to increasing our understanding of bacterial diversity.

(1)




\section{METHODS}

313 Data Acquisition and Cleaning. To conduct the analysis, 12,602 genome sequences labeled

314 either Escherichia or Shigella were downloaded from GenBank on 26 June, 2018 using batch

315 Entrez and the list of GCAs accession numbers from NCBI Genome database (including plasmid 316 sequences when applicable). This dataset (Supplementary Table 1) was cleaned to obtain an

317 informative and diverse set of 10,667 E. coli and Shigella genomes that captures the diversity of

318 the species as sequenced to date. In addition to the GenBank genomes, a total of 125,771 read 319 sets labeled as either E. coli or Shigella were downloaded from the SRA database. After cleaning 320 the dataset, we utilized $\mathrm{Mash}^{28}$, a program that approximates similarity between two genomes in 321 nucleotide content, and an in-house Python script to create a matrix of distances for all 10,667 322 genomes. This matrix was then clustered using hierarchical clustering after converting the Mash 323 distance to a Pearson's Correlation Coefficient distance to ensure that clustering results were

324 based on a genome's overall similarity to the whole species.

325 To evaluate the quality of the data set, various sequence quality scores were calculated as 326 described $^{29}$ by Land et al.. Following the recommended quality score cutoff value of 0.8 , the 327 dataset was filtered to include only genomes with a total quality score of 0.8 or higher. Applying 328 the same cutoff value to the sequence quality score alone resulted in an extremely restricted 329 dataset that no longer addressed the goals of this study. Genome size was restricted to greater 330 than $3 \mathrm{Mb}$ and less than $6.77 \mathrm{Mb}$ to remove questionably sized genomes, which could be due to 331 contamination or modified genomes that are not representative of the natural E. coli species. 332 After applying these two steps, 10,855 genomes remained in the assembled genome dataset for 333 analysis.

334 To further clean the dataset, we filtered genomes that were outside the statistical distribution of 335 Mash distances within the dataset. Assuming that Shigella species are all members of E. coli, we 336 decided to use type strains for the Escherichia and Shigella genera (accession numbers 337 GCA_000613265.1 and GCA_002949675.1, respectively) to quickly filter the set of 10,855 338 genomes for erroneous or low-quality genomes that may have slipped through the previous 339 cleaning steps. The Mash values of the 10,855 genomes compared to each type strain were 
340 broken into percentiles ranging from $10 \%$ to $99.995 \%$. A cutoff percentile of $98.5 \%$ was

341 determined to provide sufficient cleaning without risking a large loss of data (data not shown)

342 and was applied to each type strain Mash value set. Genomes that were found in both sets after

343 filtering were retained to produce the final dataset of 10,667 genomes.

344 Microreact. Microreact ${ }^{16}$, was utilized to visualize the resultant clustering of the Mash data as 345 this provides an easy and fast medium to further explore the results of the analysis. To leverage

346 the search capabilities of Microreact, we mapped metadata found for our dataset from the 347 database PATRIC ${ }^{30}$ (downloaded on 2019/6/20). This allows the exploration of our results using 348 a number of shared characteristics and queries such as "geographic location" or "serovar" that 349 although outside the scope of the current study, could be used as a topic for future analyses to 350 increase our understanding of E. coli species.

351 Mash and Clustering Analysis. Genetic distances between all 10,667 genomes were calculated 352 using 'mash dist' with a k-mer size of 21 and a sampling size of 10,000. The resulting output was 353 converted into a distance matrix with assembly accession numbers as columns and rows. To 354 improve the clustering results and to provide a standard metric that allows comparison of 355 different analytical methods, we converted the Mash distance value into a similarity measure via 356 the Pearson correlation coefficient ${ }^{31}$. This returns values ranging from -1 (total negative linear 357 correlation) to 1 (total positive linear correlation), where 0 is no linear correlation. Since 358 clustering-based methods require a distance measure, the values were subtracted from 1 to 359 convert them into a distance measure. These distance measures were then clustered in $\mathrm{R}$ using 360 'hclust' and the 'ward.D2' method. A clustered heatmap was generated using the hclust 361 dendrogram to reorder the rows and columns of the distance matrix within the heatmap, while 362 values from the raw distance matrix of Mash distances were mapped to color. To determine the 363 height to cut the hclust dendrogram and to accurately predict phylogroups that optimally 364 overlapped with existing phylogroups, we compared multiple different cutoff values and 365 methods to obtain cutoff values. Taking the maximum height present in the hclust dendrogram 366 and multiplying it by $1.25 \times 10^{-2}$ was found to provide both accurate predictions and a standard method that scales with the data supplied. Sufficient accuracy was defined by the cutoff at which 
368 the last literature accepted phylogroup was visible, in this case representing the $\mathrm{C}$ phylogroup 369 splitting off from B1. Some detailed results of both the cutoff percentile and hclust height testing 370 are included for 10,667 genomes in Supplementary Table 5.

371 Medoid selection for species representation. Using the Mash values for the entire species, a 372 medoid was defined for each phylogroup. The medoid is the "real" center of the phylogroup, as it 373 has to exist within the dataset, and was chosen as the genome that has the lowest average 374 distance to all other genomes in its phylogroup. We subsequently tested if one genome from each 375 of the phylogroups would be enough to accurately classify any given genome sequence claimed 376 to be E. coli or Shigella. The 'aggregate' function of R was used to find the mean across each 377 phylogroup. Isolating each phylogroup, reclustering, and calculating the medoid did not yield as 378 accurate results as calculating the medoid per phylogroup with respect to the entire 10,667 379 genome dataset.

Addition of SRA reads. The keywords "Escherichia coli" and "Shigella" filtered with "DNA"

381 for biomolecule and "genome" for type was used to retrieve SRA IDs from the NCBI SRA database on March 22, 2019. For large scale data transfer, these SRA genomes were downloaded using the high throughput file transfer application Aspera (http://asperasoft.com). To ease computational and organizational load, the 125,771 read sets obtained from the SRA were divided into five subsets of different sequencing technologies: 3 Illumina paired read sets, 1 mixed technology with paired reads, and 1 mixed technology with single reads. The 5 sets of reads were then converted from fastq to fasta format to be processed by Mash using a python script which removed all non-sequence data from the fastq file.

The SRA sequence reads were sketched using Mash (v2.1) and the same k-mer and sketch sample size as the 10,667 dataset. This version change was due to the addition of read pooling in the read mode which automatically joins paired reads, eliminating the need to concatenate or

392 otherwise process paired read sets. All read sets were sketched individually so that read sets that 393 caused an error when sketching were dropped from the analysis before sketching. A total of 39423,680 raw reads could not be sketched. The $-\mathrm{m}$ setting was set to 2 to decrease noise in the 395 sketches of the reads. After sketching the reads within the subsets, all sketches were 
396

397

398

399

400

401

402

403

404

405

406

407

408

409

410

411

412

413

414

415

416

417

418

419

420

421

422

423

concatenated into a sketch for that subset using the paste command of Mash. The concatenated sketch of each subset was then compared to the 14 medoids using Mash dist. As all five subsets had the same reference, the distance output from each subset was concatenated to one file. This single SRA distance output file was then analyzed to evaluate the quality of the SRA dataset. Due to how distances are calculated, Mash can consistently flag genomes of very low quality since the major basis of a Mash value is how many hits are present out of sketches sampled. The top 5 most numerous distances of the SRA read sets corresponded to 0 to 4 hits of the possible 10,000 sketches per genome. This indicates the presence of extremely low-quality samples within the SRA dataset. A histogram of the SRA Mash distance results was created to analyze the distribution of Mash distances of the entire 102,091 SRA dataset (results not shown). A final Mash distance cutoff of 0.04 was chosen based on the maximum Mash value in the 10,667 whole set that was 0.0393524 . Although this low cutoff might potentially eliminate useful information, it insured quality of the SRA dataset. This retained 95,525 reads that had at least one Mash distance to a phylogroup medoid within the chosen cutoff.

The distance output was transferred into a matrix with reads as columns and rows containing a phylogroup medoid. For each read the smallest Mash distance to a medoid was identified, and the corresponding medoid noted (Supplementary Table 3). We then created a distance matrix from the Mash distance output of the 95,525 reads that met the above cutoff with reads as rows and medoids as columns. Due to computational load this distance matrix was loaded into Python 3 instead of R. A clustered heatmap was made using Seaborn, Matplotlib, and Scipy with the 'clustermap' function. Instead of clustering both rows and columns, columns (phylogroups) were ordered the same as Fig. 1 and rows were sorted as follows: number of hits to phylogroups (ascending $=$ True) and Mash distance (ascending $=$ False). This provided a quick visualization method for the SRA dataset with a consistent sorting criterion to make comparison between Fig. 2c and the Supplemental heatmaps much easier.

Cytoscape visualization. The Mash distance matrix of the 10,667 genomes was filtered to include only the 14 medoids along the columns. This filtered matrix was transformed into a new 3 column matrix where the first column contains the identifier for a genome to be compared to 
424 the medoid present in the second column. The third column contains the Mash value for that 425 pairwise comparison. A sliding cutoff ranging from 0.04 to 0.0095 with increments of 0.005 was 426 applied to the Mash value column and rows with values above the sliding cutoff for an iteration 427 were removed. These data tables were imported into Cytoscape (version 3.7.1) with the first 428 column as the source node and the medoid column as the target node. The Prefuse Force 429 Directed Weighted layout was then applied to the network with the Mash distance serving as the 430 weight. Phylogroup membership was mapped with a metadata table and colors were assigned 431 based on the colors used in Fig. 1. For each cutoff the resultant graph was output as an SVG. All 432 SVGs were then compiled into a video to ease visualization of the Cytoscape graphs.

433 Statistical analysis of genome sizes and percent GC content. Genome sizes and percent of GC 434 content was calculated using the 'infoseq' package from EMBOSS suite v6.6.0.0. A dataframe 435 with sequence ID, percentage of GC content, genome size, and phylogroup ID was made. 436 Library 'ggplot2' from R was used to plot genome sizes and GC content. Library 'dplyr' from R 437 was used to perform analysis of Variance ANOVA test and Tukey HSD tests. The homogeneity 438 of variances was tested using Levene's test and the normality assumption of the data was 439 checked using Shapiro-Wilk test. As some of the groups didn't meet the criteria of the 440 assumption of normality, Kruskal-Wallis test was performed as well as non-parametric 441 alternative to one-way ANOVA. Kruskal-Wallis test rejected both null hypothesis (means of 442 genome size or percent of GC content are similar between the different phylogroups), with p443 value $<2.2 \mathrm{e}^{-16}$ in both cases. Raw results from these tests are available in Supplementary Table 4445.

445 Pangenome analyses and clustering. All 10,667 genomes were reannotated using Prokka ${ }^{32}$ 446 v1.13, with parameters: --rnammer --kingdom Bacteria --genus Escherichia -species coli --gcode 447 11. All protein-coding sequences $(n=51,400,905)$ were clustered using UCLUST from 448 USEARCH $^{33}$ v.10.0.240 into protein families using cut-off values of $80 \%$ of protein sequence 449 similarity, $80 \%$ of query sequence coverage, e-value equal or less than 0.0001 (parameters 450 evalue 0.0001 -id 0.8 -query_cov 0.8, with maxaccepts 1 and maxrejects 8). For the core genome 451 various inclusion percentages were compared, since we included draft genomes existing in 
452 multiple contigs. The optimum was defined that allowed 3\% omissions, giving a species core 453 genome defined as those genes present in $97 \%$ of the genome collection. Therefore, protein 454 families with presence in at least $97 \%$ of the total set strains were considered part of the core 455 genome of $E$. coli species.

456 The pan- and core genome for each of the 14 phylogroups were then separately clustered using 457 the same cut-off parameters as the entire set at species level.

459 MLST analysis. The sequence type for all 10,667 assembled genomes was assessed using the 460 program "mlst” version 2.18.0 from Seemann T, Github: https://github.com/tseemann/mlst, 461 using both the Achtman and Pasteur MLST schemas for E. coli from PubMLST website 462 (https://pubmlst.org/) developed ${ }^{34}$ by Keith Jolley. Results were collected and are accessible in 463 our microreact database: https://microreact.org/project/10667ecoli/b4431cf8

464 Core genome matrix creation and visualization. Core genome clusters for the 14 phylogroups 465 obtained using UCLUST v.10.0.240 in the previous analysis were used again with UCLUST 466 v.10.0.240 using the same parameters to find the intersection of core genes between the core 467 clusters of the 14 phylogroups. A binary matrix with cluster ID as column labels, genome IDs as 468 row names, and the number of genes belonging to that cluster as the cell value was constructed 469 using the main output from UCLUST. This matrix was then supplied to an "in house" python 470 script that sorts the pangenome matrix such that the gene clusters found in all phylogroups are 471 placed first (species' core genome). Then groups are sorted by abundance per phylogroup to 472 isolate phylogroup core genes. All leftover gene groups are sorted by phylogroup and abundance 473 and added to the end of the sorted gene cluster list. The Mash tree obtained earlier for the 10,667 474 dataset was then loaded and used to sort the order of the organisms within the sorted matrix. 475 Finally, Matplotlib was used to visualize the sorted matrix.

476 Phylogenetic analysis of core gene families. The set of core gene clusters of the 14 medoids was 477 extracted from the core genome clusters of the entire species and from them single copy ortholog 478 groups were identified to construct a phylogenomic tree. In total a set of 2,613 single gene 
479 (clusters without paralogs paralogs) ortholog groups were aligned using MAFFT ${ }^{35}$ v.7.110. The 480 model of evolution for each of the 2,613 protein clusters was calculated using IQ-TREE ${ }^{36}$ 481 v.1.6.10 with parameters -m TESTONLY -nt AUTO. Once the best model of evolution was 482 obtained for each of the core protein families, those clusters that shared model of evolution were 483 sent together to IQ-TREE for a better estimation of the substitution model parameters using -m 484 MF+MERGE, -nt AUTO and selecting the final model of evolution with mset parameter. In the last step, all partitions obtained with their corresponding model of evolution were sent again to IQ-TREE for final estimation of the phylogenetic tree for the 14 medoids using ultrafast bootstraping approach (-bb 1000). The resulted core genome tree was re-rooted using the B2-1, B2-2 and G phylogroups branch, according to the results obtained from the Mash analysis and the literature ${ }^{17}$ (Gonzalez-Alba et. Al, 2019).

490 The pangenome matrix needed as input for Count ${ }^{37}$ v10.04 for the 14 medoids was constructed 491 using UCLUST (with same parameters for pangenome calculation as in previous analyses). A 492 pivot table was built using the main output from UCLUST and pandas library in a python3 script 493 using the function 'pivot_table' with agglomeration function=sum. Count v10.04 program was 494 used for gene family expansion/contraction analysis, using an optimized gain-loss-duplicated 495 model $^{38}$ using Poisson family size distribution, 4 gamma categories for each calculation across 496 families (Edge length, Loss rate, Gain rate and Duplication rate) and different lineage specific 497 variation for gain-loss ratio and duplication-loss ratio between lineages. Measurements were 498 done using 1,000 optimization rounds (reaching convergence before the last iteration) and 0.01 499 convergence threshold on the likelihood.

Principal Coordinate Analysis. The PCoA plot in Fig. 3b was created using R, the entire 501 pangenome matrix for the 10,667 assembled genomes, and the libraries 'ade4' version 1.7-13 502 and 'labdsv' version 2.0-1. A Jaccard distance matrix of the pangenome matrix was created using 503 the 'dist.binary' function from 'ade4'. To create the PCoA data, the Jaccard distance matrix was 504 used in the 'pco' function of 'labdsv' with $\mathrm{k}=10,666$ (allowing each genome to be a unique 505 dimension). The resultant PCoA data was then graphically rendered using R 'plot' and colors 506 were added by genome classification as shown in Fig. 1. 
507 Reporting Summary. Further information on research design is available in the Nature Research

508 Reporting Summary linked to this article.

\section{Data availability}

510 The data supporting the findings of the study are available in this article, its Supplementary

511 Information files, or from the corresponding author upon request.

513 Code availability

514 Code is available on GitHub: https:/github.com/kalebabram/100k_E_coli_Project

\section{REFERENCES}

516

517 1. Jang, J. et al. Environmental Escherichia coli: ecology and public health implications-a

518 review. J. Appl. Microbiol. 123, 570-581 (2017).

519 2. Fischer Walker, C. L., Sack, D. \& Black, R. E. Etiology of Diarrhea in Older Children,

520 Adolescents and Adults: A Systematic Review. PLoS Negl. Trop. Dis. 4, e768 (2010).

521 3. Dunne, K. A. et al. Sequencing a piece of history: complete genome sequence of the original

522 Escherichia coli strain. Microb. Genom. 3, mgen000106 (2017).

523 4. Pettengill, E. A., Pettengill, J. B. \& Binet, R. Phylogenetic Analyses of Shigella and

524 Enteroinvasive Escherichia coli for the Identification of Molecular Epidemiological Markers:

525 Whole-Genome Comparative Analysis Does Not Support Distinct Genera Designation. Front.

526 Microbiol. 6,1573 (2016).

527 5. Chattaway, M. A., Schaefer, U., Tewolde, R., Dallman, T. J. \& Jenkins, C. Identification of

528 Escherichia coli and Shigella Species from Whole-Genome Sequences. J. Clin. Microbiol. 55, $529 \quad 616-623$ (2017). 
530 6. Clermont, O., Bonacorsi, S. \& Bingen, E. Rapid and Simple Determination of the Escherichia

531 coli Phylogenetic Group. Appl. Environ. Microbiol. 66, 4555-4558 (2000).

532 7. Gordon, D. M., Clermont, O., Tolley, H. \& Denamur, E. Assigning Escherichia coli strains to

533 phylogenetic groups: multi-locus sequence typing versus the PCR triplex method: MLST

534 versus Clermont method. Environ. Microbiol. 10, 2484-2496 (2008).

535 8. Tenaillon, O., Skurnik, D., Picard, B. \& Denamur, E. The population genetics of commensal

536 Escherichia coli. Nat. Rev. Microbiol. 8, 207-217 (2010).

537 9. Clermont, O., Christenson, J. K., Denamur, E. \& Gordon, D. M. The Clermont Escherichia coli

538 phylo-typing method revisited: improvement of specificity and detection of new phylo-

539 groups: A new E. coli phylo-typing method. Environ. Microbiol. Rep. 5, 58-65 (2013).

540 10. Meier-Kolthoff, J. P. et al. Complete genome sequence of DSM 30083T, the type strain

541 (U5/41T) of Escherichia coli, and a proposal for delineating subspecies in microbial

542 taxonomy. Stand. Genomic Sci. 9, 2 (2014).

543 11. Clermont, O. et al. Characterization and rapid identification of phylogroup G in Escherichia

544 coli, a lineage with high virulence and antibiotic resistance potential. Environ. Microbiol. 21,

$545 \quad 3107-3117$ (2019).

546 12. Walk, S. T. et al. Cryptic Lineages of the Genus Escherichia. Appl. Environ. Microbiol. 75,

$547 \quad 6534-6544(2009)$.

548 13. Carlos, C. et al. Escherichia coli phylogenetic group determination and its application in the

549 identification of the major animal source of fecal contamination. BMC Microbiol. 10, 161

$550 \quad$ (2010) 
551 14. Vangchhia, B. et al. Phylogenetic diversity, antimicrobial susceptibility and virulence

552 characteristics of phylogroup F Escherichia coli in Australia. Microbiology 162, 1904-1912

553 (2016).

554 15. Struyf, A., Hubert, M. \& Rousseeuw, P. Clustering in an Object-Oriented Environment. J.

$555 \quad$ Stat. Softw. 1, 1-30 (1997).

556 16. Argimón, S. et al. Microreact: visualizing and sharing data for genomic epidemiology and

557 phylogeography. Microb. Genom. 2, e000093 (2016).

558 17. Gonzalez-Alba, J. M., Baquero, F., Cantón, R. \& Galán, J. C. Stratified reconstruction of

559 ancestral Escherichia coli diversification. BMC Genomics 20, 936 (2019).

560 18. Weinert, L. A. \& Welch, J. J. Why Might Bacterial Pathogens Have Small Genomes? Trends

$561 \quad$ Ecol. Evol. 32, 936-947 (2017).

562 19. Bhunia, A. K. Escherichia coli. in Foodborne Microbial Pathogens: Mechanisms and

563 Pathogenesis (ed. Bhunia, A. K.) 249-269 (Springer New York, 2018). doi:10.1007/978-1-

564 4939-7349-1_14.

565 20. Balbi, K. J., Rocha, E. P. C. \& Feil, E. J. The Temporal Dynamics of Slightly Deleterious

566 Mutations in Escherichia coli and Shigella spp. Mol. Biol. Evol. 26, 345-355 (2009).

567 21. Sharma, V. K., Akavaram, S., Schaut, R. G. \& Bayles, D. O. Comparative genomics reveals

568 structural and functional features specific to the genome of a foodborne Escherichia coli

$569 \quad$ O157:H7. BMC Genomics 20, 196 (2019).

570 22. Udaondo, Z., Molina, L., Segura, A., Duque, E. \& Ramos, J. L. Analysis of the core genome

571 and pangenome of Pseudomonas putida. Environ. Microbiol. 18, 3268-3283 (2016). 
572 23. Abreo, E. \& Altier, N. Pangenome of Serratia marcescens strains from nosocomial and

573 environmental origins reveals different populations and the links between them. Sci. Rep. 9,

$574 \quad 1-8(2019)$.

575 24. Salipante, S. J. et al. Large-scale genomic sequencing of extraintestinal pathogenic

576 Escherichia coli strains. Genome Res. 25, 119-128 (2015).

577 25. Nicolas-Chanoine, M.-H., Bertrand, X. \& Madec, J.-Y. Escherichia coli ST131, an Intriguing

578 Clonal Group. Clin. Microbiol. Rev. 27, 543-574 (2014).

579 26. Petty, N. K. et al. Global dissemination of a multidrug resistant Escherichia coli clone. Proc.

$580 \quad$ Natl. Acad. Sci. USA 111, 5694-5699 (2014).

581 27. Lecointre, G., Rachdi, L., Darlu, P. \& Denamur, E. Escherichia coli molecular phylogeny using

582 the incongruence length difference test. Mol. Biol. Evol. 15, 1685-1695 (1998).

583 28. Ondov, B. D. et al. Mash: fast genome and metagenome distance estimation using

584 MinHash. Genome Biol. 17, 132 (2016).

585 29. Land, M. L. et al. Quality scores for 32,000 genomes. Stand. Genomic Sci. 9, 20 (2014).

586 30. Wattam, A. R. et al. Improvements to PATRIC, the all-bacterial Bioinformatics Database and

587 Analysis Resource Center. Nucleic Acids Res. 45, D535-D542 (2017).

588 31. Pearson's Correlation Coefficient. in Encyclopedia of Public Health (ed. Kirch, W.) 1090-

5891091 (Springer Netherlands, 2008). doi:10.1007/978-1-4020-5614-7_2569.

590 32. Seemann, T. Prokka: rapid prokaryotic genome annotation. Bioinformatics 30, 2068-2069

$591 \quad$ (2014).

592 33. Edgar, R. C. Search and clustering orders of magnitude faster than BLAST. Bioinformatics 26, 
2460-2461 (2010).

594

34. Jolley, K. A. \& Maiden, M. C. BIGSdb: Scalable analysis of bacterial genome variation at the population level. BMC Bioinformatics 11, 595 (2010).

35. Katoh, K. \& Standley, D. M. MAFFT Multiple Sequence Alignment Software Version 7: Improvements in Performance and Usability. Mol. Biol. Evol. 30, 772-780 (2013). 268-274 (2015).

37. Csürös, M. Count: evolutionary analysis of phylogenetic profiles with parsimony and likelihood. Bioinformatics 26, 1910-1912 (2010).

38. Csűrös, M. \& Miklós, I. Streamlining and Large Ancestral Genomes in Archaea Inferred with a Phylogenetic Birth-and-Death Model. Mol. Biol. Evol. 26, 2087-2095 (2009).

\section{ACKNOWLEDGMENTS}

607 This work was supported by NIH/NIGMS grant 1P20GM121293 and from the Helen Adams \& 608 Arkansas Research Alliance Endowment in the Department of Biomedical Informatics, College 609 of Medicine. We thank Dr. Scott Emrich for discussions about Mash in the early stages of this 610 manuscript. We also appreciate the contribution of Dr. Juan Carlos Galan and his group for 611 allowing us to adapt Fig. 3 of their manuscript recently published as Gonzalez-Alba et al., 2019 612 into Fig. 2a of our manuscript. 


\section{Author contributions}

618 K.Z.A and Z.U. conceived and designed all the experiments with help from D.W.U.

619 K.Z.A and Z.U. conducted all the experiments and drafted the manuscript with contributions 620 from all authors.

621 C.B. assisted with Cytoscape analysis.

622 V.W. assisted with the download of SRA reads.

623 T.M.W. provided advice and discussion and helped with the revision of the manuscript and 624 improvement of figures.

625 M.S.R. II provided advice, discussion, and assisted with the phylogenetic analysis as well as 626 revising the manuscript and improving figures.

627 D.W.U. conceived the work, provided funding and provided advice and discussions.

628

\section{Competing interesting}

630 Author declare no competing interests.

631

\section{Additional information}

633

634 Extended data is available for this paper at https://github.com/kalebabram/100k_E_coli_Project

635 Supplementary information is available for this paper at

636 Correspondence and request for materials should be addressed to D.W.U.

637 Reprints and permission information is available at www.nature.com/reprints

638

639

640

641

642

643

644

645 


\section{Tables}

647 Table 1. Summary of pangenome analysis results. Values obtained from the different pangenome analysis using 648 the 14 phylogroups separately and the entire set of assembled genomes (10,667 genomes) using UCLUST (Edgar, 649 2010). Same parameters were used to all the analysis.

\begin{tabular}{|c|c|c|c|c|c|c|c|c|c|c|}
\hline \multirow{2}{*}{$\begin{array}{c}\text { Phylogroup } \\
\text { All }\end{array}$} & \multicolumn{2}{|c|}{$\begin{array}{r}\text { Core genome } \\
(97 \% \text { strains) } \\
\text { clusters proteins }\end{array}$} & \multicolumn{2}{|c|}{$\begin{array}{l}\text { Accessory genome } \\
\text { clusters proteins }\end{array}$} & \multicolumn{2}{|c|}{$\begin{array}{l}\text { Unique } \\
\text { ters proteins }\end{array}$} & \multicolumn{2}{|c|}{$\begin{array}{l}\text { Total (Pan genome) } \\
\text { clusters proteins }\end{array}$} & $\begin{array}{l}\text { Core/pan } \\
(\%) \\
\text { clusters }\end{array}$ & $\begin{array}{l}\text { No. of } \\
\text { strains }\end{array}$ \\
\hline & 2,663 & $28,566,052$ & 82,821 & $22,783,754$ & $4 \quad 50,499$ & 51,099 & 135,983 & $51,400,905$ & 1.96 & $6 \quad 10,667$ \\
\hline $\mathbf{A}$ & 3,184 & $7,142,893$ & 41,769 & $3,246,591$ & 24,501 & 24,828 & 69,454 & $10,414,312$ & 4.58 & $8 \quad 2,232$ \\
\hline B1 & 3,141 & $9,365,646$ & 44,019 & $4,887,086$ & $6 \quad 24,590$ & 24,844 & 71,750 & $14,277,576$ & 4.38 & 2,960 \\
\hline B2-1 & 3,708 & $2,016,812$ & 10,990 & 619,867 & $7 \quad 7,048$ & 7,180 & 21,746 & $2,643,859$ & 17.05 & 541 \\
\hline B2-2 & 3,425 & $4,709,983$ & 22,762 & $1,819,538$ & 12,566 & 12,763 & 38,753 & $6,542,284$ & 8.84 & $4 \quad 1,367$ \\
\hline C & 3,899 & $2,132,258$ & 10,413 & 738,879 & 5,242 & 5,290 & 19,554 & $2,876,427$ & 19.94 & 540 \\
\hline D1 & 3,666 & $1,006,271$ & 10,012 & 318,372 & 7,659 & 7,770 & 21 & $1,332,413$ & 17.18 & 273 \\
\hline D2 & 3,524 & 626 & 11 , & 22 & 6 , & 7,181 & 21 & 07 & 16.02 & 177 \\
\hline D3 & 3,754 & 668,359 & 7,252 & 20 & 24,814 & 4,936 & 15,820 & 587 & 23.73 & 177 \\
\hline E1 & 3,151 & 885,018 & 14,883 & 471,354 & 7,969 & 8,088 & 26,003 & $1,364,460$ & 12.12 & 279 \\
\hline E2(0157) & 4,060 & $3,080,073$ & 6,128 & 743,413 & 4,442 & 4,535 & 14,630 & $3,828,021$ & 27.75 & 750 \\
\hline $\mathbf{F}$ & 3,486 & 698,031 & 9,465 & 288,420 & 5,381 & 5,480 & 18,332 & 991,931 & 19.02 & 199 \\
\hline $\mathbf{G}$ & 3,783 & 365,756 & 5,716 & 98,269 & 4,016 & 4,066 & 13,515 & 468,091 & 27.99 & 96 \\
\hline Shig1 & 3,128 & 564,868 & 4,903 & 256,426 & 2,815 & 2,883 & 10,846 & 824,177 & 28.84 & 177 \\
\hline Shig2 & 3,732 & $3,383,814$ & 6,870 & 719,247 & 4,751 & 4,799 & 15,353 & $4,107,860$ & 24.31 & 899 \\
\hline
\end{tabular}

650

651 Legends of Tables

652 Table 1. Summary of pangenome analysis results. Values obtained from the different 653 pangenome analysis using the 14 phylogroups separately and the entire set of assembled 654 genomes (10,667 genomes) using UCLUST (Edgar, 2010). Same parameters were used to all the 655 analysis

\section{Legends of Figures}

657 Fig. 1. Heatmap representation of 10,667 genomes using Mash distances. The color bars at

658 the top of the heatmap identify the phylogroups as predicted from the analysis. The scale to the 659 left of the dendrogram corresponds to the resultant cluster height of the entire dataset obtained 660 from hclust function in R. The colors in the heatmap are based on the pairwise Mash distance 661 between the genomes. Teal colors represent similarity between genomes with the darkest teal 
662 corresponding to identical genomes reporting a Mash distance of 0. Brown colors represent low 663 genetic similarity per Mash distance, with the darkest brown indicating a maximum distance of 664 0.039. Genomes of relative median genetic similarity have the lightest color.

665 Fig. 2. Summary of phylogroup differentiation and heatmap representation of sequence 666 reads from the SRA database. a, Evolutionary scenario in the diversification of E. coli adapted 667 from Gonzalez-Alba et. al, 2019 based on their methodology "SP-mPH", a combination of 668 "stratified phylogeny" and "molecular polymorphism hallmark". Each branch reflects SNPs 669 accrued by each phylogroup over time. Branch length is not proportional to the observed 670 evolutionary distance. b, Summary of the Cytoscape analysis. Phylogroups are colored based on

671 the same colour scheme as Fig. 1. Phylogroups with more than one member are gray coloured.

672 The Mash distance that each division occurs at is indicated by numerical value in the gray bar 673 that runs down the side of this panel. c, Clustered heatmap of 91,261 sequnce reads. The heatmap 674 colors are based on the pairwise Mash distance between the SRA read sets and the 14 medoid 675 genomes of each phylogroup, which are presented in the same order as in Fig. 1. To be included, 676 SRA reads sets had to have 3 or more medoid comparisons producing a Mash distance equal to 677 or less than 0.04 . This removed 4,264 SRA read sets from the dataset. The number of SRA reads 678 mapped to each medoids is given below the heatmap. Supplementary Fig. 2 contains additional 679 cut-offs ranging from one to 14 phylogroups.

680 Fig. 3. Pangenome representations of $\boldsymbol{E}$. coli and Shigella. A. Each bar length of the circular 681 bar plot represents the total number of proteins of a single genome, grouped by phylogroup. The 682 proteins belonging to the ${ }^{\mathrm{TOT}}$ core $_{97}$ genome are shown in green. Additional proteins shared in 683 each ${ }^{\mathrm{PHY}}$ core $_{97}$ genome are shown in blue, while purple is reserved for accessory proteins. B. 684 Principal Coordinate Analysis plot of 135,983 protein families of 10,667 assembled genomes. 685 Phylogroups are indicated by the same color scheme used in Figs. 1 and 2. C. Core genome 686 matrix of 6,719 phylogroup core clusters and 10,667 assembled genomes. Clusters are sorted 687 such that the core for the species is placed first, then the phylogroup core genes are placed sorted 688 by their overall abundance in the species for each phylogroup in the same order as Fig. 1, finally 689 the remaining clusters are placed by overall abundance. Phylogroup unique core genes are 690 indicated by purple blocks which do not appear in other phylogroups. 
691 Fig. 4. Phylogenetic representations of $E$. coli species using the core genome of the 14 692 medoids. A. The tree was built using a set of 2,613 core clusters with no paralogs using IQ693 TREE (Nguyen et al., 2015). B. Summary representation of Count output. The phylogenetic tree 694 presents the different gain/loss/duplication ratios obtained per each phylogroup as output of 695 Count v.10.04 software (Csürös, 2010). Dots in branches represent "informative ellipsis" where 696 the length of the undotted section of the branch multiplied by the inverse ratio of undotted 697 section is equal to the true rate of the branch. For example, assuming the displayed branch length 698 is 1 and $1 / 10^{\text {th }}$ of the branch is solid then the true rate of the branch would be 10 . 699 Gain/loss/duplication rates for each branch are shown in the table.

\section{Supplementary Information}

701

702

703

704

705

706

707

708

709

710

711

\section{Supplementary Figures}

713 Supplementary Figure 1. Distribution of Shigella genomes over phylogroups.

714 Supplementary Figure 2. Heatmaps of all SRA reads that had a Mash score of at least 0.04 to 715 one medoid. Each heatmap has a set of genomes with at least the indicated number of hits to a 716 medoid of at least 0.04 .

717 Supplementary Figure 3. Violin-plots of the distribution of genome size (A) and genomic GC 718 content (B) by phylogroup. Bar-plots inside the violins represent values for mean and mean plus 719 one standard deviation per phylogroup. Phylogroups that have values significantly different to all 
bioRxiv preprint doi: https://doi.org/10.1101/708131; this version posted January 15,2020 . The copyright holder for this preprint (which was not certified by peer review) is the author/funder, who has granted bioRxiv a license to display the preprint in perpetuity. It is made available under aCC-BY-NC-ND 4.0 International license.

720 other phylogroups (according to F statistics test) are marked with a red asterisk.

721 Supplementary Figure 4. Cut-offs for core genome calculation. Core genomes established at a

722 cutoff of $90 \%$ to $100 \%$ per phylogroup. Last section represents the rate of cluster drop-off

723 between percentages ( $90 \%$ to $99 \%$ )

724 


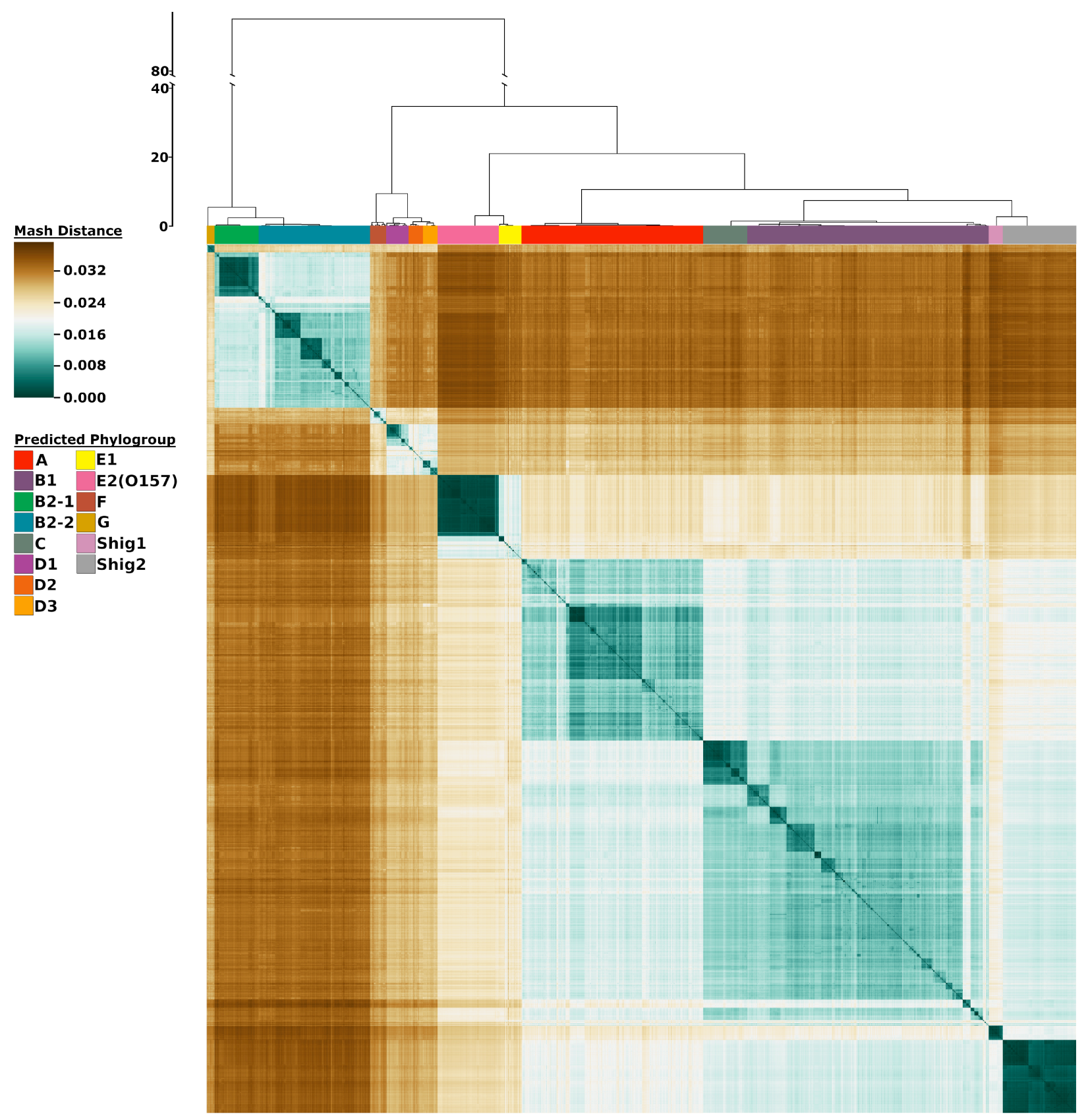

726 Fig. 1. Heatmap representation of 10,667 genomes using Mash distances. The color bars at

727 the top of the heatmap identify the phylogroups as predicted from the analysis. The scale to the

728 left of the dendrogram corresponds to the resultant cluster height of the entire dataset obtained

729 from hclust function in R. The colors in the heatmap are based on the pairwise Mash distance

730 between the genomes. Teal colors represent similarity between genomes with the darkest teal 
731 corresponding to identical genomes reporting a Mash distance of 0. Brown colors represent low

732 genetic similarity per Mash distance, with the darkest brown indicating a maximum distance of

733 0.039. Genomes of relative median genetic similarity have the lightest color.

734

735

736

737

738

739

740

741

742

743

744

745

746

747

748

749 

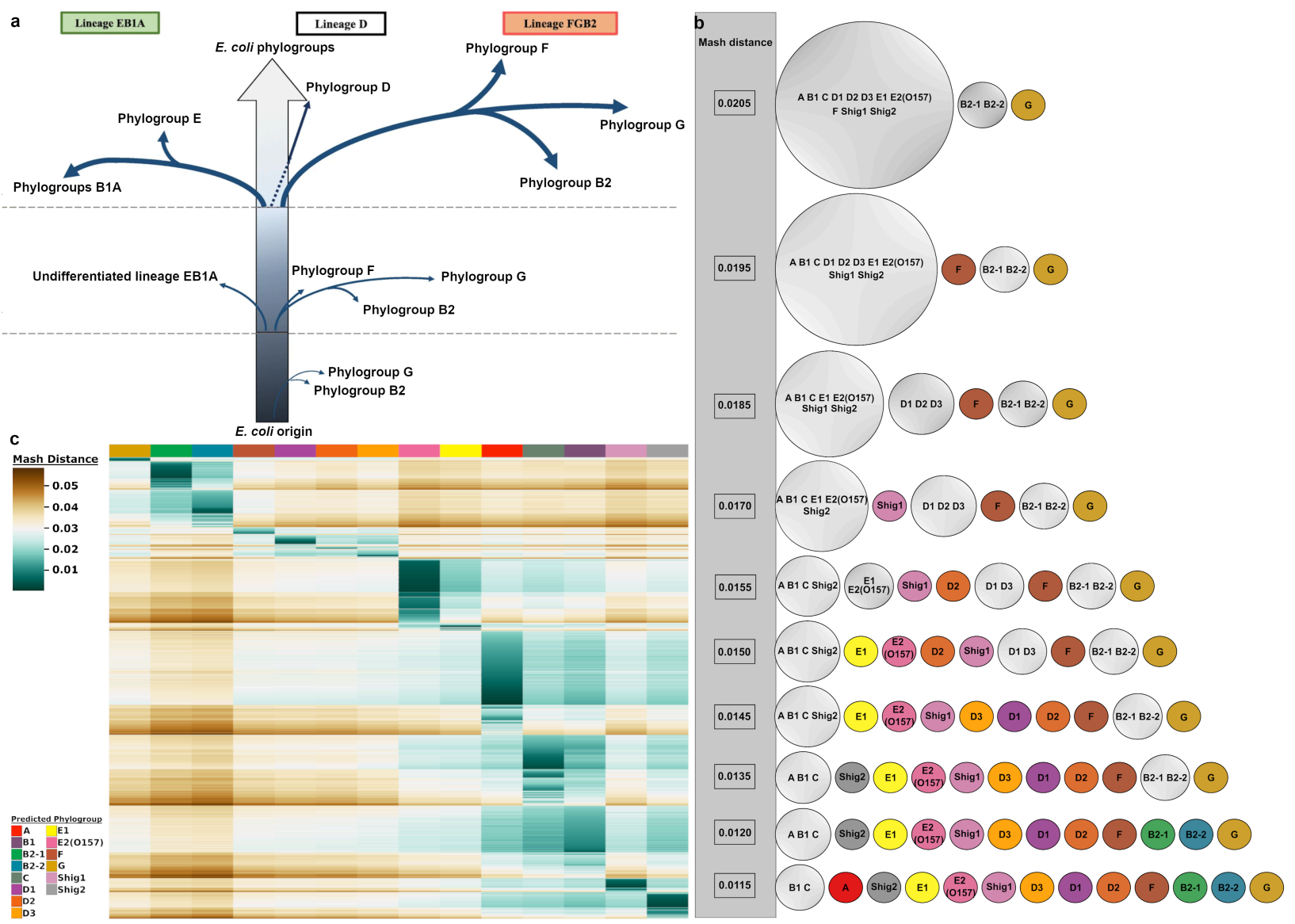

751 Fig. 2. Summary of phylogroup differentiation and heatmap representation of sequence from Gonzalez-Alba et. al, 2019 based on their methodology "SP-mPH", a combination of "stratified phylogeny" and "molecular polymorphism hallmark". Each branch reflects SNPs accrued by each phylogroup over time. Branch length is not proportional to the observed evolutionary distance. b, Summary of the Cytoscape analysis. Phylogroups are colored based on the same colour scheme as Fig. 1. Phylogroups with more than one member are gray coloured.

758 The Mash distance that each division occurs at is indicated by numerical value in the gray bar that runs down the side of this panel. c, Clustered heatmap of 91,261sequnce reads. The heatmap colors are based on the pairwise Mash distance between the SRA read sets and the 14 medoid genomes of each phylogroup, which are presented in the same order as in Fig. 1. To be included,

762 SRA reads sets had to have 3 or more medoid comparisons producing a Mash distance equal to 763 or less than 0.04 . This removed 4,264 SRA read sets from the dataset. The number of SRA reads 
bioRxiv preprint doi: https://doi. org/10.1101/708131· this version posted January 15,2020 . The copyright holder for this preprint (which was not certified by peer review) is the author/funder, who has granted bioRxiv a license to display the preprint in perpetuity. It is made available under aCC-BY-NC-ND 4.0 International license.

764 mapped to each medoids is given below the heatmap. Supplementary Fig. 2 contains additional

765 cut-offs ranging from one to 14 phylogroups.

766

767

768

769

770

771

772

773

774

775

776

777

778

779

780

781

782 
bioRxiv preprint doi: https://doi.org/10.1101/708131; this version posted January 15,2020 . The copyright holder for this preprint (which was not certified by peer review) is the author/funder, who has granted bioRxiv a license to display the preprint in perpetuity. It is made available under aCC-BY-NC-ND 4.0 International license.
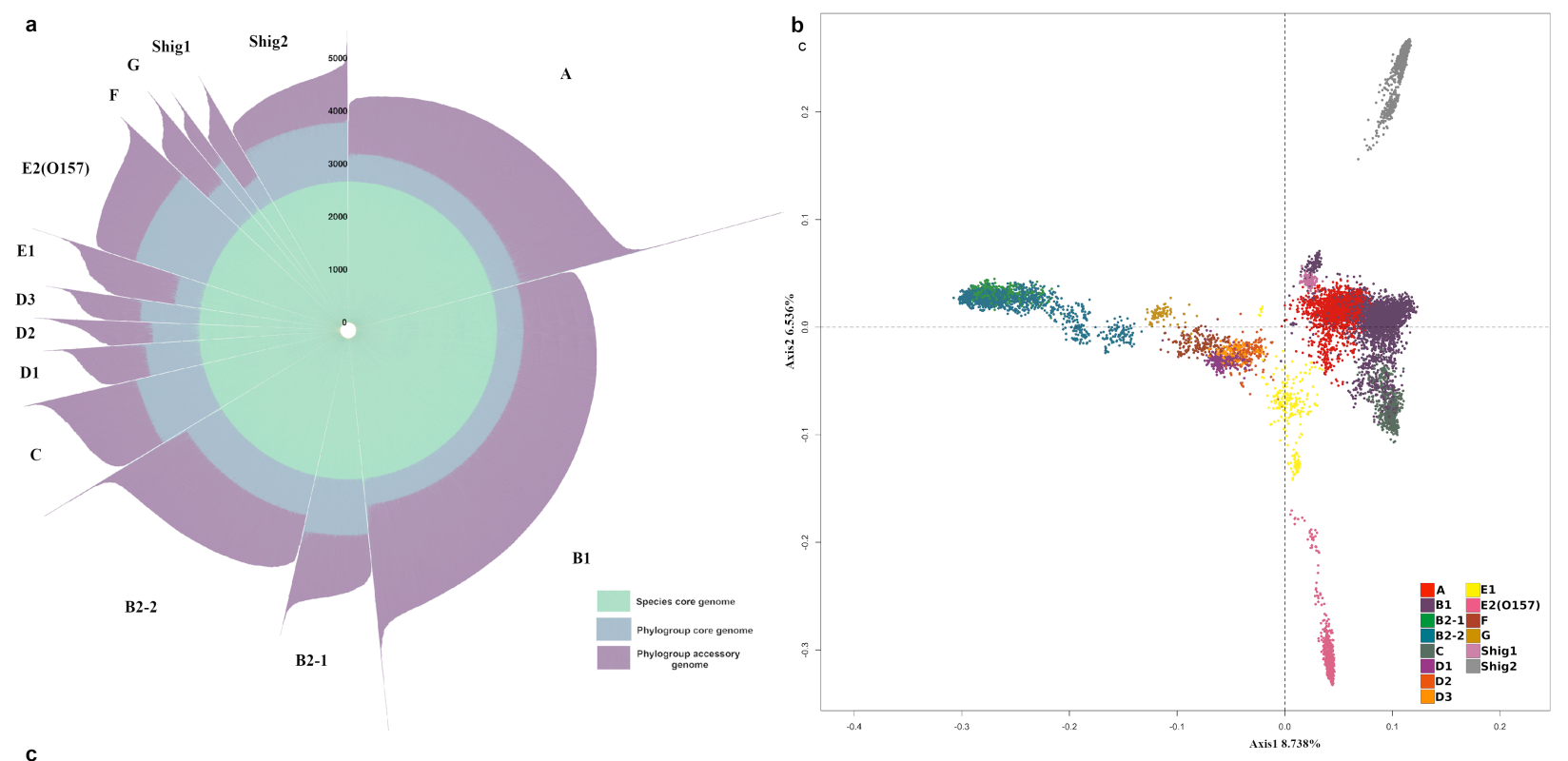

c

Mash Tree $(\mathbf{1 0 , 6 6 7}$ strains) Core genome matrix
$\mathbf{6 , 7 1 9}$ clusters

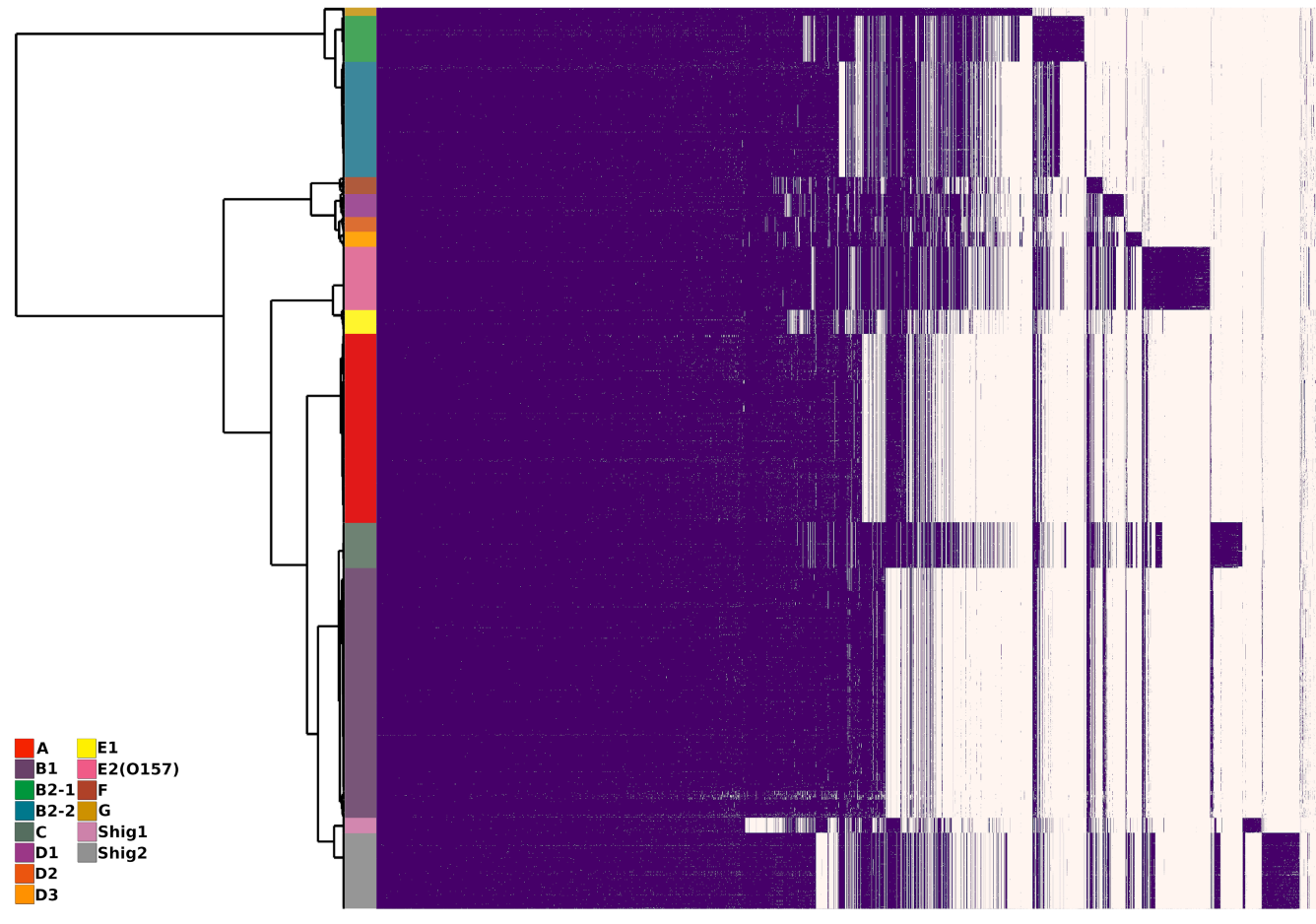

784 Fig. 3. Pangenome representations of $\boldsymbol{E}$. coli and Shigella. A. Each bar length of the circular 785 bar plot represents the total number of proteins of a single genome, grouped by phylogroup. The 786 proteins belonging to the ${ }^{\text {TOT }}{ }^{-}{ }{ }_{97}$ genome are shown in green. Additional proteins shared in 787 each ${ }^{\mathrm{PHY}}$ core97 genome are shown in blue, while purple is reserved for accessory proteins. B. 
788 Principal Coordinate Analysis plot of 135,983 protein families of 10,667 assembled genomes.

789 Phylogroups are indicated by the same color scheme used in Figs. 1 and 2. C. Core genome

790 matrix of 6,719 phylogroup core clusters and 10,667 assembled genomes. Clusters are sorted

791 such that the core for the species is placed first, then the phylogroup core genes are placed sorted

792 by their overall abundance in the species for each phylogroup in the same order as Fig. 1, finally

793 the remaining clusters are placed by overall abundance. Phylogroup unique core genes are

794 indicated by purple blocks which do not appear in other phylogroups.

795

796

797

798

799

800

801

802

803

804

805

806

807

808 

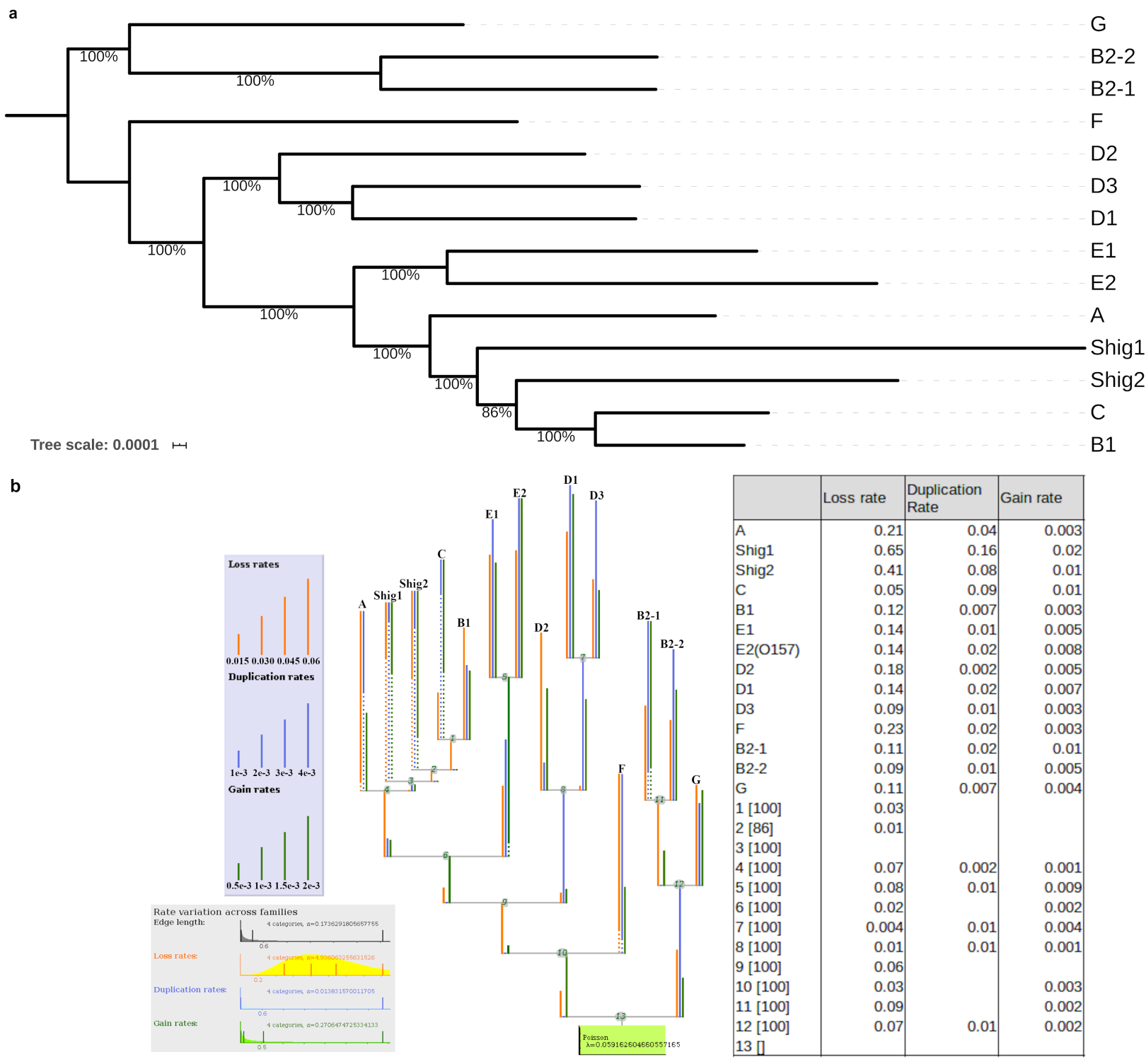

810 Fig. 4. Phylogenetic representations of $E$. coli species using the core genome of the 14

811 medoids. A. The tree was built using a set of 2,613 core clusters with no paralogs using IQ-

812 TREE (Nguyen et al., 2015). B. Summary representation of Count output. The phylogenetic tree

813 presents the different gain/loss/duplication ratios obtained per each phylogroup as output of

814 Count v.10.04 software (Csürös, 2010). Dots in branches represent "informative ellipsis" where

815 the length of the undotted section of the branch multiplied by the inverse ratio of undotted 816 section is equal to the true rate of the branch. For example, assuming the displayed branch length 817 is 1 and $1 / 10^{\text {th }}$ of the branch is solid then the true rate of the branch would be 10 . 
bioRxiv preprint doi: https://doi org/10.1101/708131 t this version posted January 15,2020 . The copyright holder for this preprint (which was not certified by peer review) is the author/funder, who has granted bioRxiv a license to display the preprint in perpetuity. It is made available under aCC-BY-NC-ND 4.0 International license.

818 Gain/loss/duplication rates for each branch are shown in the table.

819 